Nonlinearity of Concreteness and Semantic Depth: The Logistic Curve

Baijayanta Roy

Independent Scholar

Author Note

Baijayanta Roy declares that he has no conflict of interest. 


\begin{abstract}
Abstractness of a concept is viewed from two broad perspectives. Concreteness - the amount of sensory information in a concept and Precision - the amount of general information present in a concept. Though Concreteness and Precision is known to have been two different ways of measuring abstractness, the relationship between the two is not clear. This study proposes that the Mean Concreteness of words in a given Depth range (a measure of Precision) has a nonlinear relationship with Depth and can be modelled by a Generalized Logistic Function. The justification of the asymptotical upper and lower limit of Mean Concreteness in the Logistic model is based on the assumption of the ineffability of sensory information and embodied cognition. The proposed mathematical description based on generalized logistic function showed a good fit for the data collected from Brysbaert Dataset $\left(\mathrm{R}^{2}=0.939\right)$ and was fairly accurate in predicting the Mean Concreteness scores in MRC psycholinguistic Dataset $\left(\mathrm{R}^{2}=0.911\right)$.

Keywords: Concreteness, Precision, Depth, Nonlinearity, Logistic Function, Sensory Information.
\end{abstract}


Nonlinearity of Concreteness and Semantic Depth: The Logistic Curve

Abstraction is the cognitive process of decontextualizing an object of interest by identifying a set of 'invariant central characteristics' (Burgoon et al., 2013, p. 502). This is thought to be at the very core of language and communication. Therefore, abstractness enjoys an important role in psychology, anthropology, and linguistics and computer science. Abstractness in natural language has proven to be of immense importance and a tough case for automated systems to crack, e.g. in word sense disambiguation (Kwong, 2008), identification of metaphoric language (Neuman et al., 2013), automatic translation (Zhu et al., 2010) etc. In psychology, the level of abstractness (or concreteness) of a concept has been shown to have significant correlations with cognitive performance (Kroll \& Merves, 1986; Paivio, 1968; Walker \& Hulme, 1999), social attitudes (Murray et al., 1996; Sherman et al., 2012), learning and memory (Kreitler et al., 1983; Medin et al., 1983), creativity (Förster et al., 2004; Liberman et al., 2012) and even with preservation of self-esteem (Maass, 1999).

\footnotetext{
ABSTRACTNESS

To understand abstraction, it would seem only necessary to be able to objectively measure abstractness of a concept, category or a word. Researchers have long relied on manipulations that induce abstractness in subject's thought process to study its effects; see Burgoon et al. (2013) for review. Others have systematically measured abstractness associated with concepts and words by simply asking participants to rate them based on how abstract/concrete it feels (Brysbaert et al., 2014; Paivio, Yuille, \& Madigan, 1968; Scott et al., 2019; Wilson, 1988). However, a big hurdle in achieving a purely objective measure of abstractness is the vagueness of its definition (Barsalou, 2003; Burgoon et al., 2013; Pollock,
} 
2018). Recently, a number of novel techniques have been developed to compute abstractness of a concept using hierarchical semantic networks such as WordNet and BabelNet (Colla et al., 2018; Iliev \& Axelrod, 2017). Although these methods have significantly automated the process of determining abstractness of a concept, they are not entirely objective because the hierarchy of the semantic networks (such as WordNet) are themselves based on the opinions of a handful of scholars.

\section{Measuring Abstractness.}

Underlying these methods are two ways of looking at abstractness. According to the first perspective, lesser the amount of sensory information encoded in a concept, the more abstract it is. This way of looking at abstractness was adopted by experimenters who were interested to capture people's intuition of abstractness in their ratings, (e.g. Brysbaert et al., 2014; Paivio et

al., 1968; Spreen \& Schulz, 1966). As Pollock (2018) noted, this way of capturing abstractness of a concept is essentially negative, in that it is only concerned about the opposite of abstractness, i.e. Concreteness. And what makes a concept concrete is the presence of sensory information. According to the second perspective, lesser the amount of information encoded in a concept, the more abstract it is. This approach is inspired by the idea that an abstract concept is more inclusive (Brown, 1958; Rosch et al., 1976). If concepts can be arranged in a hierarchy on the basis of IS-A relationships (e.g. a Labrador IS-A dog and a dog IS-A mammal) then the most inclusive concepts would be towards the top of that hierarchy. The more inclusive a concept is; the lesser amount of details it contains. Therefore, the less abstract a concept is the more information it contains. Iliev and Axelrod (2017, p. 716) called this measure - Precision. The authors also mention two ways of measuring precision: $P$-inclusiveness and $P$-depth, where $\mathrm{P}$ inclusiveness of a concept $\mathrm{c}$, that has $\mathrm{n}$ number of direct and indirect descendants in a semantic 
hierarchy (e.g. WordNet) containing a total of $\mathrm{N}$ concepts, is measured by: $-\log _{2}((n+1) / N)$. P-depth on the other hand is simply the number of concepts it takes in a IS-A hierarchy to go from a word to the root of the semantic tree. For WordNet the root of the tree (and hence the most abstract word) is the word - 'entity' (Miller, 1995).

\section{The Paradox.}

If concreteness and precision are both measuring the same phenomenon, namely abstractness, they should show significant correlations in their measurements. But this is clearly not the case as shown by Iliev and Axelrod (2017, p. 723). Not only concreteness and precision measures (both P-depth and P-inclusiveness) were weakly correlated, P-inclusiveness and Pdepth are themselves found to be weakly correlated. Iliev and Axelrod (2017, p. 727) conclude that, although intuitively concreteness and precision are broadly looking at the same thing, they are measuring two different aspects of abstractness, and point out that the main difference between the two perspectives is that the former (concreteness) cares about the amount of sensory information while the later (precision) cares about the amount of general information. While this is a valuable insight, it doesn't explain the relationship between concreteness and precision.

\section{Ineffability of Sensory Information.}

I believe that the relationship between concreteness and precision can be understood by shedding light on our limitations of codifying sensory vs. general information. While the presence of sensory information encoded in a concept helps cognition, a.k.a. concreteness effect, sensory information is simply not as abundantly available or encodable as we might think (Levinson \& Majid, 2014; Majid \& Levinson, 2011). For example, olfactory senses are long known to be hard to describe in English (Wnuk \& Majid, 2014). And though visual modality is known to be the most effable in English (Winter, Perlman, \& Majid, 2018), it has its limits too. 
For example, we find it easy to describe abstract geometrical shapes but find it very hard to codify people's faces in words. And it is not because of the limitations of a particular language but because of our cognitive architecture (Levinson \& Majid, 2014, p. 417). Just like no amount of language can help a congenially blind person understand 'red', our direct experience is limited by our modalities of senses and hence we have a limited pool of direct encodable sensory information. This is overcome by our indirect ways of understanding things that are beyond our senses (such as UV Rays). As a result we end up developing concepts that code much less sensory information (e.g. electromagnetic waves, frequency etc.) but can have a lot of abstract information. Therefore, while we can keep on encoding more and more general information in concepts, the amount of sensory information will have to come to a saturation after a while. In other words, it is reasonable to assume that concreteness and precision will have a nonlinear relationship such that, after a rapid increase the average concreteness scores will stop increasing significantly with respect to precision scores.

The purpose of this study is to mitigate the paradox of abstraction, pointed out by Iliev and Axelrod (2017), by exploring the nonlinearity between concreteness and precision. As mentioned earlier, precision can be computed in two ways, namely P-inclusiveness and P-depth. This study will restrict its focus on the relationship only between concreteness and P-depth (henceforth mentioned as Depth). A mathematical model representing the relationship between concreteness and depth will be fitted on the concreteness data by Brysbaert et al. (2014) and would be tested to predict Mean Concreteness scores in MRC psycholinguistic dataset (Wilson, 1988). 


\section{DATA}

\section{Parts of Speech.}

In this study, concreteness scores of only common nouns were used to calculate depth. The reason is twofold. Firstly, the assumption made previously regarding the limitation of the codability of sensory information with respect to general information has ontological implications. Although there is no reason why this argument cannot be extended to verbs and other parts of speech, the assumption straightforwardly applies to common nouns and makes the interpretation a little simpler. Secondly, in the gradualist viewpoint of language evolution scholars give primacy to nouns. Heine and Kuteva (2002), in their layering model of the evolution of grammar put nouns in the first layer upon which verbs, adjectives etc. were hypothesized to evolve in a cumulative manner. Also Givón (2002) believes that since grammatical categories are more abstract and complex than lexical categories, it is only reasonable to assume that languages evolved from a one-word-stage which were primarily comprised of nouns. Since nouns play a fundamental role in our ontology, restricting ourselves to only nouns is not only simpler but can be revealing.

\section{Concreteness Scores.}

In this study the following standardized psycholinguistic datasets were used that are available in public domain. First, Brysbaert Dataset (BRYS) is a list of concreteness ratings of 40,000 English words. About 20-30 subjects were asked to rate each word, based on how well they can experience the word directly through the five senses, on a 5-point Likert scale (Brysbaert et al., 2014). The ratings were then averaged for each word. The resultant score is a continuous variable ranging from 1 to 5 . After extracting all common nouns from the database by using part-of speech (pos) tagging in NLTK, the dataset was reduced to 14,593 words. In this 
study, the BRYS dataset is used to develop the proposed model and generate estimates of its parameters. Secondly, the MRC psycholinguistic database (Wilson, 1988) is a huge collection that contains concreteness scores of 150,837 words. The scale differs from BRYS in that it ranges from 100-700. Due to technical limitation, it was only possible to extract 3480 common nouns manually from their official website. The concreteness scores of these nouns were then converted to BRYS scale. In this study, the MRC dataset is used to test how well the proposed logistic model can predict mean concreteness scores. Third, dataset of 750 nouns by Troche et al. (2017) contains ratings of 14 cognitive dimensions such as color, emotion, valence, polarity, motion, space etc. This dataset was used to compare how the behavior of sensory information (color, taste-smell, auditory etc.) differs from non-sensory information (morality, thought etc.) with respect to semantic Depth.

\section{Conversion of MRC to BRYS Scale.}

To check how well our model, developed on BRYS dataset, predicts mean concreteness scores of MRC database, it was necessary to convert MRC concreteness scores $\left(\mathrm{C}_{\mathrm{MRC}}\right)$, that range from 100-700 to BRYS concreteness ( $\left.\mathrm{C}_{\mathrm{BRYS}}\right)$ scale ranging from 1-5. Solving linear equations with two boundary conditions $\left(\mathrm{C}_{\mathrm{MRC}}=100, \mathrm{C}_{\mathrm{BRYS}}=1\right.$ and $\left.\mathrm{C}_{\mathrm{MRC}}=700, \mathrm{C}_{\mathrm{BRYS}}=5\right)$ is simply not reasonable because there is no guarantee that these equalities hold psychologically in the participants' minds while scoring. Therefore a linear regression model was run on the nouns present in both the datasets $(\mathrm{N}=2724)$ to test the relationship between their $\mathrm{C}_{\mathrm{BRYS}}$ (dependent) and $\mathrm{C}_{\mathrm{MRC}}$ (independent). The regression analysis showed significant linear relationship between the two $(F(1,2722)=16562.910, p<0.001)$ with adjusted $\mathrm{R}^{2}=0.859$. The predicted parameters were found to be: intercept $=77.159(\mathrm{t}=24.235, \mathrm{p}<0.001)$ and slope $=104.143(\mathrm{t}=128.697, \mathrm{p}$ 
$<0.001)$. Using these parameters the concreteness scores of 2724 nouns in MRC database were converted into BRYS scale by the following relationship:

$$
C_{\text {MRCconverted }}=\frac{C_{M R C}-77.159}{104.143}
$$

Where, $C_{\text {MRCconverted }}$ is the converted MRC concreteness scores in BRYS scale.

\section{Depth Scores.}

The Depth Score of a word is calculated using WordNet 3.1 implemented in NLTK (version 3.4.5) using python. This is the number of hypernyms required for a word to reach the most inclusive word in WordNet, which is 'entity' (coded as 'entity.n.01' in WordNet). Since a given word can have more than one meaning (polysemous words), Depth scores of all the meanings (known as 'synsets' in WordNet) were calculated and averaged to get a single Depth Score for a given word. This method for calculating semantic depth was also used by Iliev and Axelrod (2017, p. 721). The Depth scores computed for nouns extracted from BRYS dataset $($ min value $=1, \max$ value $=19)$ were chopped into 100 bins starting from 1 with a resulting width of 0.180 for each bin.

\section{MODEL AND RESULTS.}

\section{Nonlinearity.}

A simple scatter plot does not reveal a visually discernible relationship between concreteness and depth. However, if we chop the independent variable (Depth) into small bins and then scatter plot the mean concreteness scores in each bin, a pattern emerges (Figure 1). As is quite apparent, as Depth increases the Mean Concreteness scores, expectedly, stop increasing after a while. A similar trend can be observed in the concreteness scores in MRC 
psycholinguistic dataset (Figure 2). The 750 word dataset by Troche et al. (2017) has controlled ratings of a number of cognitive dimensions such as thought, morality, color, auditory, tactile etc. associated with each word. The cognitive dimensions that are sensory in nature e.g. color, auditory, taste-smell, tactile show a logistic trend, indicating an asymptotic upper limit (Figure 3). On the contrary, the cognitive dimensions that are decidedly not sensory in nature, such as thought, morality and social dimensions, they show the exact opposite trend (Figure 4). This reinforces the conviction that the average sensory information in concepts systematically tends to saturate asymptotically to an upper limit and is not a random behavior.

\section{Model.}

Since a clear pattern is recognizable between mean concreteness and binned depth, this can be assumed to be the manifestation of a logistic function. However, the asymptotic limits are not obviously intuitive and in need of some justification. First, the asymptotic lower limit can be justified by the realization that every semantic concept have a certain amount of sensory information associated with it, i.e., the mean concreteness scores is ideally never zero. This justification is founded in the theory of embodied/grounded cognition (Barsalou, 1999) which has enjoyed wide empirical support in recent times (Glenberg \& Kaschak, 2002; Pecher et al., 2003), see Pecher et al. (2011) for review. The idea is, whenever we think of something (say apple) our brain reactivates and simulates those neural sensory-motor pathways that were formed during the experience of that thing (apple). Even the most abstract concepts such as freedom and independence are shown to be grounded on image schemas embodied in experiences related to different modalities (Pecher et al., 2011). Therefore it seems reasonable to assume that even the 
most abstract words will be associated with some level of concreteness, however miniscule, due to its embodiment in sensory-motor mechanism.

Secondly, as discussed earlier, the asymptotic upper limit to the amount of how much concrete information is encodable is based on the idea of ineffability of sensory information. It might seem that experts such as artists, musicians, master chefs etc. with their keen sense of sensory stimulus may push the limits of how much sensory information can be encoded in concepts. But studies have shown that coffee and wine tasters have a domain specific advantage and use more source based descriptors such as 'oakey' and 'nutty' (as opposed to evaluative descriptors such as 'nice') to communicate smell and taste (Croijmans \& Majid, 2016). This means, that having a keen sense of smell does not necessarily add new lexical concepts loaded with more sensory information. However languages do vary in their capabilities of expressing certain modality of senses, e.g. Japanese have a $5^{\text {th }}$ taste descriptor (other than sweet, sour, salty and bitter) called umami, the taste of monosodium glutamate or 'Ajinomoto' (O'Mahony \& Ishii, 1986). Though no single hierarchy exits in terms of codability of different modalities, there are common trends. For example, vision lexicon is consistently rich and smell lexicon is almost universally poor (Majid et al., 2018). These limitations are therefore bound to be reflected in the mean concreteness scores forming an asymptotic upper bound of sensory information codability.

Thus, we should be able to model the relationship between mean concreteness scores and binned depth scores by a generalized logistic function widely popular in biology, economics, demography and linguistics:

$$
\bar{C}(d)=A+\frac{K-A}{1+e^{-r(d-M)}}
$$


Where, $\bar{C}$ is the mean concreteness in the depth bin $d, A=$ lower asymptote (the minimum $\bar{C}$ ), $K=$ upper asymptote (the maximum $\bar{C}$ ), $r=$ growth rate (the amount of sensory information per unit general information), $M=$ value of $d$ where $\bar{C}$ is $\frac{K+A}{2}$.

The parameters of the above equation were estimated by minimizing sum of squared residuals using Levenberg-Marquardt estimation method on Brysbaert dataset $(\mathrm{N}=14593)$ in SPSS. Both the sum of square convergence and parameter convergence were set to $10^{-8}$. This method requires initial values to be fed in order to get an estimation (Cavallini, 1993). The initial values, eyeballed from Figure 1 , were taken to be $\mathrm{K}=4.5, \mathrm{~A}=2.7, \mathrm{M}=50$ and $\mathrm{r}=0.05$. The analysis yielded parameters shown in Table 1. A linear regression found a significant association between the estimated and observed mean concreteness scores where, $F(1,14590)=225717.454$, $\mathrm{p}<0.001$ and $\mathrm{R}^{2}=0.939$ with a Standard Error of Estimate $=0.09889$ in the BRYS dataset (Figure 5). Judging by the $\mathrm{R}^{2}$ values (Table 2), this model proves to be a better fit than other traditional nonlinear models. The same analysis done with a few different set of initial values resulted in the same parameter estimates.

\section{Testing the Model.}

The concreteness scores in MRC dataset $(\mathrm{N}=3480)$, ranging from 100-700, were converted to BRYS scale, ranging from 1-5, using equation (1). The model was then tested to predict the converted mean concreteness scores $(\bar{C})$ from binned depth $(d)$. A Linear Regression between the predicted and observed Mean Concreteness scores of MRC (converted to BRYS scale) found a significant correlation where $\mathrm{F}(1,3475)=35443.075, \mathrm{p}<0.001$ and Adjusted $\mathrm{R}^{2}=$ 0.911 and standard error of estimate $=0.14283$ (Figure 6). 


\section{CONCLUSION}

From this study it is clear that concreteness and semantic depth does not have a linear relationship at least for common nouns in English. The mean concreteness and semantic depth can best be modelled by a logistic function under the assumption that the mean concreteness score has an upper and lower limit to which it asymptotically converges. The nonlinearity seems to stem from the fact that sensory information is limited in terms of cognitive availability and processing (Figure 5 and 6). The model was fairly successful in predicting the mean concreteness scores of nouns in MRC database.

\section{DISCUSSION AND SCOPE}

Since the logistic model has been used to predict the mean concreteness scores for a given range of depth scores, it by no mean implies that the upper limit of the concreteness scores themselves is set by the upper asymptote of the logistic curve. It is quite apparent from the error bars representing \pm 2 standard deviation (Figure 1 and 2 ) that the individual concreteness scores go well beyond the upper asymptote. The ineffability of sensory information should therefore be taken as a general trend, applicable over averages and not indicative of any strict limitation. Besides, the jury is still out on the issue whether the limitation of codability of sensory information is a property of some languages (language with a lower case ' 1 ') or is it a property of Language in general (with an upper case 'L') (Levinson \& Majid, 2014). The author believes that the upper limit of mean concreteness suggested in the logistic model is indicative of a trend true for Language in general and not a property of any special language. This makes the claim all the more falsifiable and would be interesting to see it unfold, which is beyond the scope of current study. 
Since, this study has been conducted with a limited scope, further tests over larger samples of concreteness scores and multiple databases are required to extend its conclusion. The logistic relation could also be tested for non-noun words, p-inclusiveness (another measure of precision), and hierarchical semantic networks of non-English languages. 


\section{References}

Barsalou, L. W. (1999). Perceptual symbol systems. Behavioral and Brain Sciences, 22(4), 577660.

Barsalou, L. W. (2003). Abstraction in perceptual symbol systems. Philosophical Transactions of the Royal Society of London. Series B: Biological Sciences, 358(1435), 1177-1187.

Brown, R. (1958). How shall a thing be called? Psychological Review, 65(1), 14.

Brysbaert, M., Warriner, A. B., \& Kuperman, V. (2014). Concreteness ratings for 40 thousand generally known English word lemmas. Behavior Research Methods, 46(3), 904-911.

Burgoon, E. M., Henderson, M. D., \& Markman, A. B. (2013). There are many ways to see the forest for the trees: A tour guide for abstraction. Perspectives on Psychological Science, $8(5), 501-520$.

Cavallini, F. (1993). Fitting a Logistic Curve to Data. The College Mathematics Journal, 24(3), 247-253. https://doi.org/10.1080/07468342.1993.11973540

Colla, D., Mensa, E., Porporato, A., \& Radicioni, D. P. (2018). Conceptual Abstractness: from Nouns to Verbs. 5th Italian Conference on Computational Linguistics, CLiC-It 2018, 70-75. CEUR Workshop Proceedings.

Croijmans, I., \& Majid, A. (2016). Not all flavor expertise is equal: The language of wine and coffee experts. PLoS One, 11(6).

Förster, J., Friedman, R. S., \& Liberman, N. (2004). Temporal construal effects on abstract and concrete thinking: consequences for insight and creative cognition. Journal of Personality and Social Psychology, 87(2), 177.

Givón, T. (2002). Bio-linguistics: the Santa Barbara lectures. John Benjamins Publishing. 
Glenberg, A. M., \& Kaschak, M. P. (2002). Grounding language in action. Psychonomic Bulletin \& Review, 9(3), 558-565.

Heine, B., \& Kuteva, T. (2002). On the Evolution of Grammatical Forms. The Transition to Language, 2, 376.

Iliev, R., \& Axelrod, R. (2017). The paradox of abstraction: Precision versus concreteness. Journal of Psycholinguistic Research, 46(3), 715-729.

Kreitler, S., Zigler, E., \& Kreitler, H. (1983). The effects of memory and abstractive integration on children's probability learning. Child Development, 1487-1496.

Kroll, J. F., \& Merves, J. S. (1986). Lexical access for concrete and abstract words. Journal of Experimental Psychology: Learning, Memory, and Cognition, 12(1), 92.

Kwong, O. O. Y. (2008). A preliminary study on the impact of lexical concreteness on word sense disambiguation. Proceedings of the 22nd Pacific Asia Conference on Language, Information and Computation, 235-244.

Levinson, S. C., \& Majid, A. (2014). Differential ineffability and the senses. Mind \& Language, 29(4), 407-427.

Liberman, N., Polack, O., Hameiri, B., \& Blumenfeld, M. (2012). Priming of spatial distance enhances children's creative performance. Journal of Experimental Child Psychology, 111(4), 663-670.

Maass, A. (1999). Linguistic intergroup bias: Stereotype perpetuation through language. Advances in Experimental Social Psychology, 31, 79-122.

Majid, A., \& Levinson, S. C. (2011). The senses in language and culture. The Senses and Society, $6(1), 5-18$.

Majid, A., Roberts, S. G., Cilissen, L., Emmorey, K., Nicodemus, B., O’grady, L., ... Cansler, B. 
L. (2018). Differential coding of perception in the world's languages. Proceedings of the National Academy of Sciences, 115(45), 11369-11376.

Medin, D. L., Dewey, G. I., \& Murphy, T. D. (1983). Relationships between item and category learning: Evidence that abstraction is not automatic. Journal of Experimental Psychology: Learning, Memory, and Cognition, 9(4), 607.

Miller, G. A. (1995). WordNet: a lexical database for English. Communications of the ACM, $38(11), 39-41$.

Murray, S. L., Holmes, J. G., \& Griffin, D. W. (1996). The benefits of positive illusions: Idealization and the construction of satisfaction in close relationships. Journal of Personality and Social Psychology, 70(1), 79.

Neuman, Y., Assaf, D., Cohen, Y., Last, M., Argamon, S., Howard, N., \& Frieder, O. (2013). Metaphor identification in large texts corpora. PloS One, 8(4).

O’Mahony, M., \& Ishii, R. (1986). A comparison of English and Japanese taste languages: Taste descriptive methodology, codability and the umami taste. British Journal of Psychology, $77(2), 161-174$.

Paivio, A. (1968). A factor-analytic study of word attributes and verbal learning. Journal of Verbal Learning and Verbal Behavior, 7(1), 41-49.

Paivio, A., Yuille, J. C., \& Madigan, S. A. (1968). Concreteness, imagery, and meaningfulness values for 925 nouns. Journal of Experimental Psychology, 76(1p2), 1.

Pecher, D., Boot, I., \& Van Dantzig, S. (2011). Abstract concepts: Sensory-motor grounding, metaphors, and beyond. In Psychology of learning and motivation (Vol. 54, pp. 217-248). Elsevier.

Pecher, D., Zeelenberg, R., \& Barsalou, L. W. (2003). Verifying different-modality properties for 
concepts produces switching costs. Psychological Science, 14(2), 119-124.

Pollock, L. (2018). Concepts and concreteness in psycholinguistics. UCL (University College London).

Rosch, E., Mervis, C. B., Gray, W. D., Johnson, D. M., \& Boyes-Braem, P. (1976). Basic objects in natural categories. Cognitive Psychology, 8(3), 382-439.

Scott, G. G., Keitel, A., Becirspahic, M., Yao, B., \& Sereno, S. C. (2019). The Glasgow Norms: Ratings of 5,500 words on nine scales. Behavior Research Methods, 51(3), 1258-1270.

Sherman, J. W., Allen, T. J., \& Sacchi, D. L. M. (2012). Stereotype confirmation and disconfirmation. Guilford Press.

Spreen, O., \& Schulz, R. W. (1966). Parameters of abstraction, meaningfulness, and pronunciability for 329 nouns. Journal of Verbal Learning and Verbal Behavior, 5(5), 459468.

Troche, J., Crutch, S. J., \& Reilly, J. (2017). Defining a conceptual topography of word concreteness: clustering properties of emotion, sensation, and magnitude among 750 english words. Frontiers in Psychology, 8, 1787.

Walker, I., \& Hulme, C. (1999). Concrete words are easier to recall than abstract words: Evidence for a semantic contribution to short-term serial recall. Journal of Experimental Psychology: Learning, Memory, and Cognition, 25(5), 1256.

Wilson, M. (1988). MRC psycholinguistic database: Machine-usable dictionary, version 2.00. Behavior Research Methods, Instruments, \& Computers, 20(1), 6-10.

Winter, B., Perlman, M., \& Majid, A. (2018). Vision dominates in perceptual language: English sensory vocabulary is optimized for usage. Cognition, 179, 213-220.

Wnuk, E., \& Majid, A. (2014). Revisiting the limits of language: The odor lexicon of Maniq. 
Cognition, 131(1), 125-138.

Zhu, Z., Bernhard, D., \& Gurevych, I. (2010). A monolingual tree-based translation model for sentence simplification. Proceedings of the 23rd International Conference on Computational Linguistics, 1353-1361. Association for Computational Linguistics. 
Tables

Table 1

Parameters of Logistic Function Estimated from BRYS Dataset

\begin{tabular}{llll}
\hline Parameters & Estimate & Standard Error & $95 \%$ Confidence Interval \\
\hline $\mathrm{K}$ & 4.129 & 0.003 & $4.122-4.135$ \\
$\mathrm{~A}$ & 2.645 & 0.007 & $2.622-2.639$ \\
$\mathrm{M}$ & 41.270 & 0.071 & $41.132-41.408$ \\
$\mathrm{r}$ & 0.147 & 0.001 & $0.144-0.150$ \\
\hline
\end{tabular}


Table 2

Goodness of Fit for various Traditional Nonlinear Models Estimated on BRYS Dataset.

\begin{tabular}{llllllllll}
\hline \multicolumn{1}{l}{ Model Summary } & \multicolumn{7}{c}{ Parameter Estimates } \\
\hline Equation & $\mathrm{R}^{2}$ & $\mathrm{~F}$ & $\mathrm{DF} 1$ & $\mathrm{DF} 2$ & $\mathrm{Sig}$ & Constant & $\mathrm{B} 1$ & $\mathrm{~B} 2$ & $\mathrm{~B} 3$ \\
\hline Linear & 0.871 & 98117.899 & 1 & 14590 & .000 & 1.927 & 0.035 & & \\
Logarithmic & 0.888 & 116004.069 & 1 & 14590 & .000 & -2.538 & 1.599 & & \\
Quadratic & 0.912 & 76043.405 & 2 & 14589 & .000 & 0.910 & 0.079 & 0.000 & \\
Cubic & 0.915 & 52498.803 & 3 & 14588 & .000 & 1.552 & 0.038 & 0.000 & $-5.318 \mathrm{E}-6$ \\
Growth & 0.851 & 83544.233 & 1 & 14590 & .000 & 0.796 & 0.010 & & \\
\hline
\end{tabular}




\section{FIGURES}

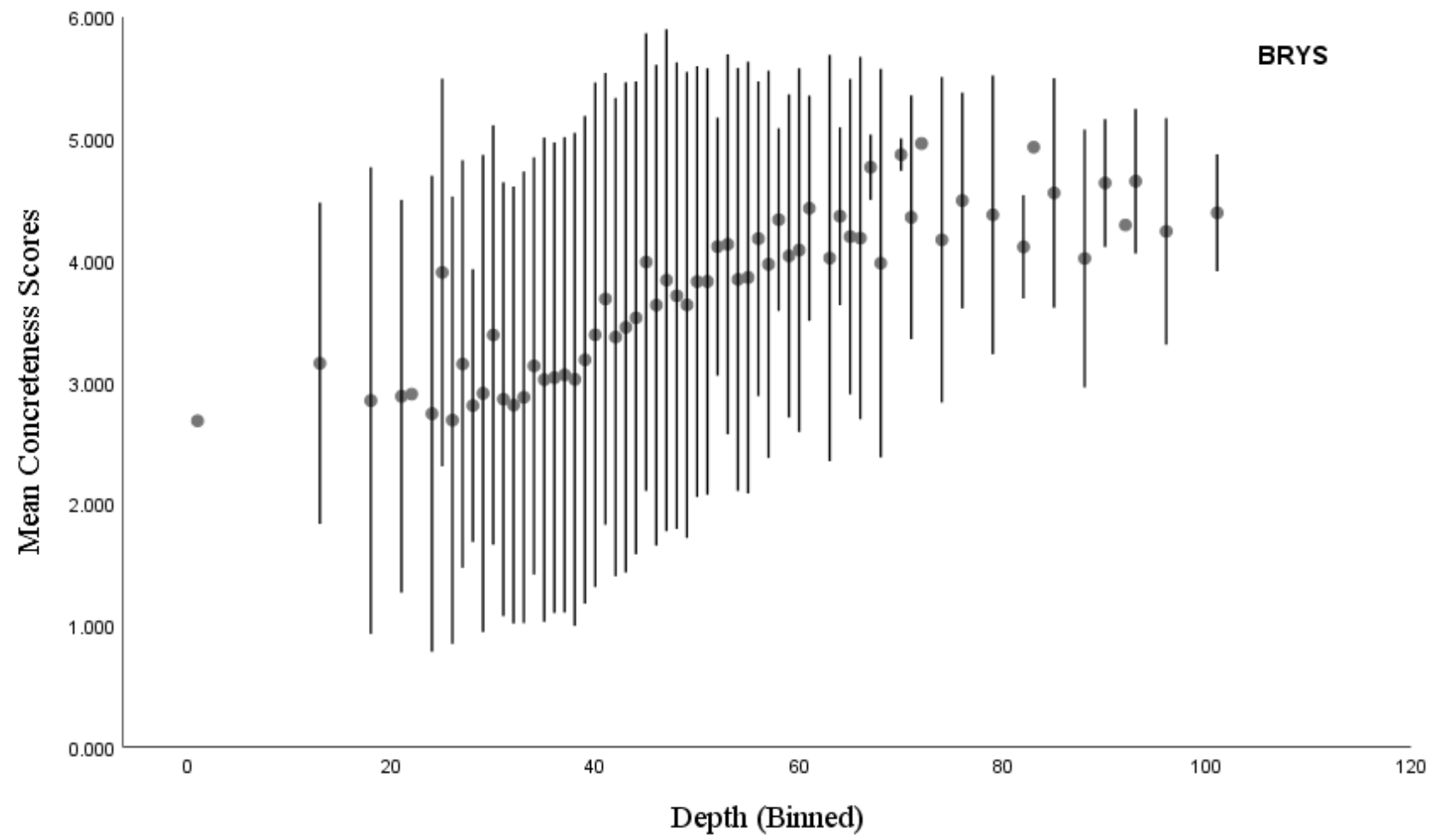

Figure 1. A Scatter Plot between Mean Concreteness Scores and Binned Depth of BRYS

Dataset. Error bars Represent \pm 2 Standard Deviation. 


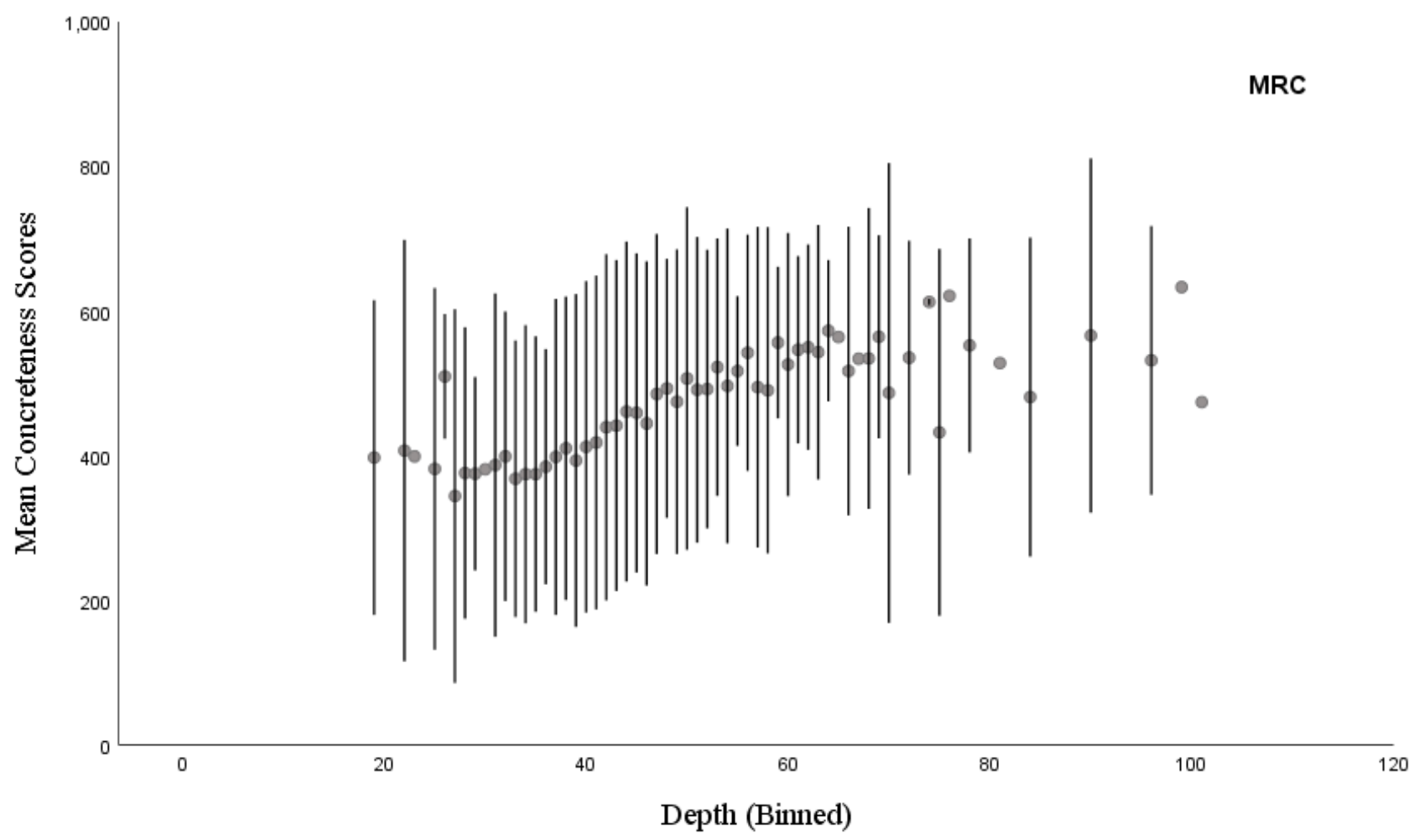

Figure 2. A Scatter Plot between Mean Concreteness Scores and Binned Depth of MRC Dataset.

Error bars Represent \pm 2 Standard Deviation. 

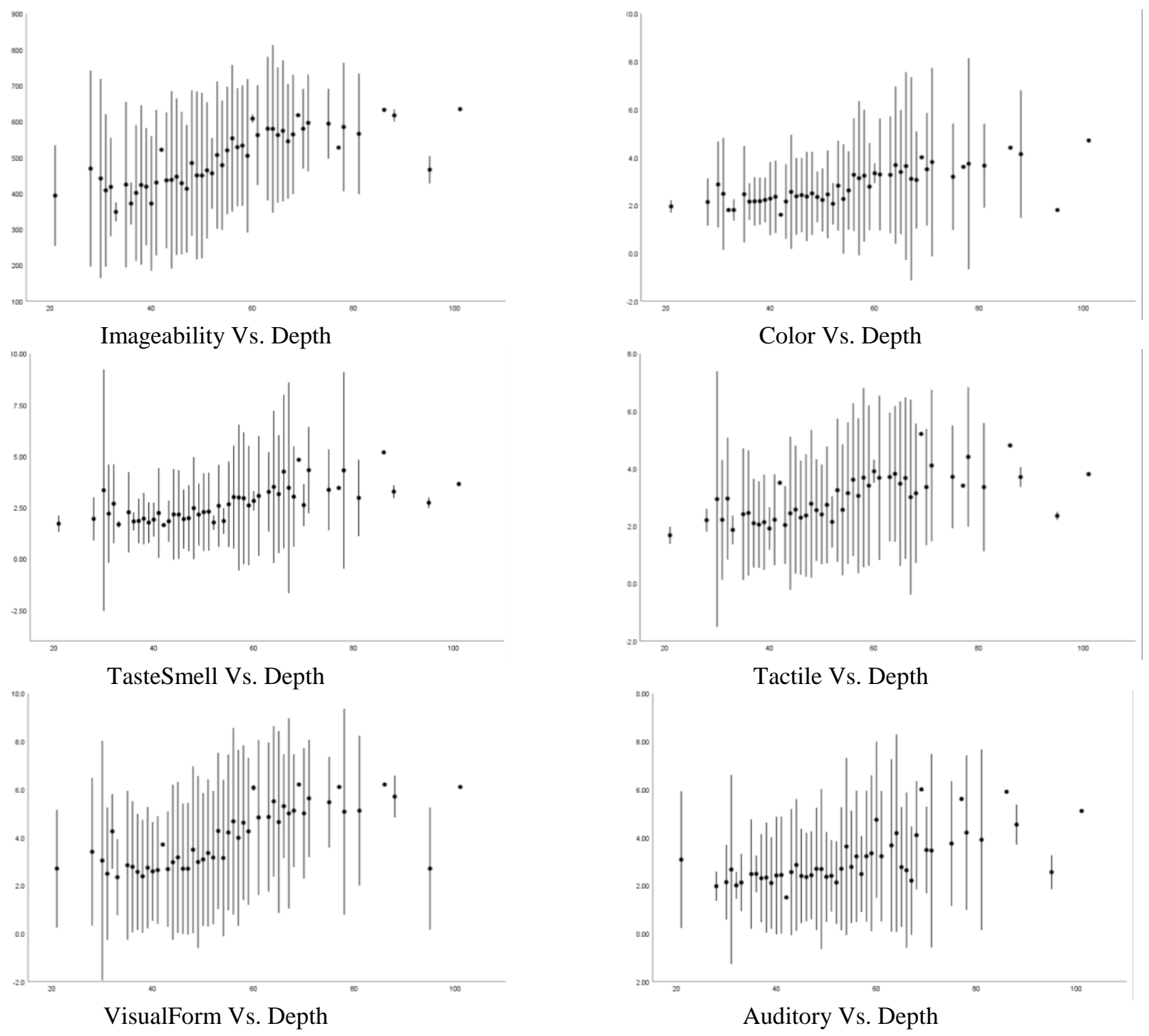

Figure 3. Scatter Plots of Mean Imageability, Color, TasteSmell, Tactile, VisualForm and Auditory measured in Crutch et al. (2013) Vs Binned Depth scores. Error bars represent \pm 2 Standard Deviation. 

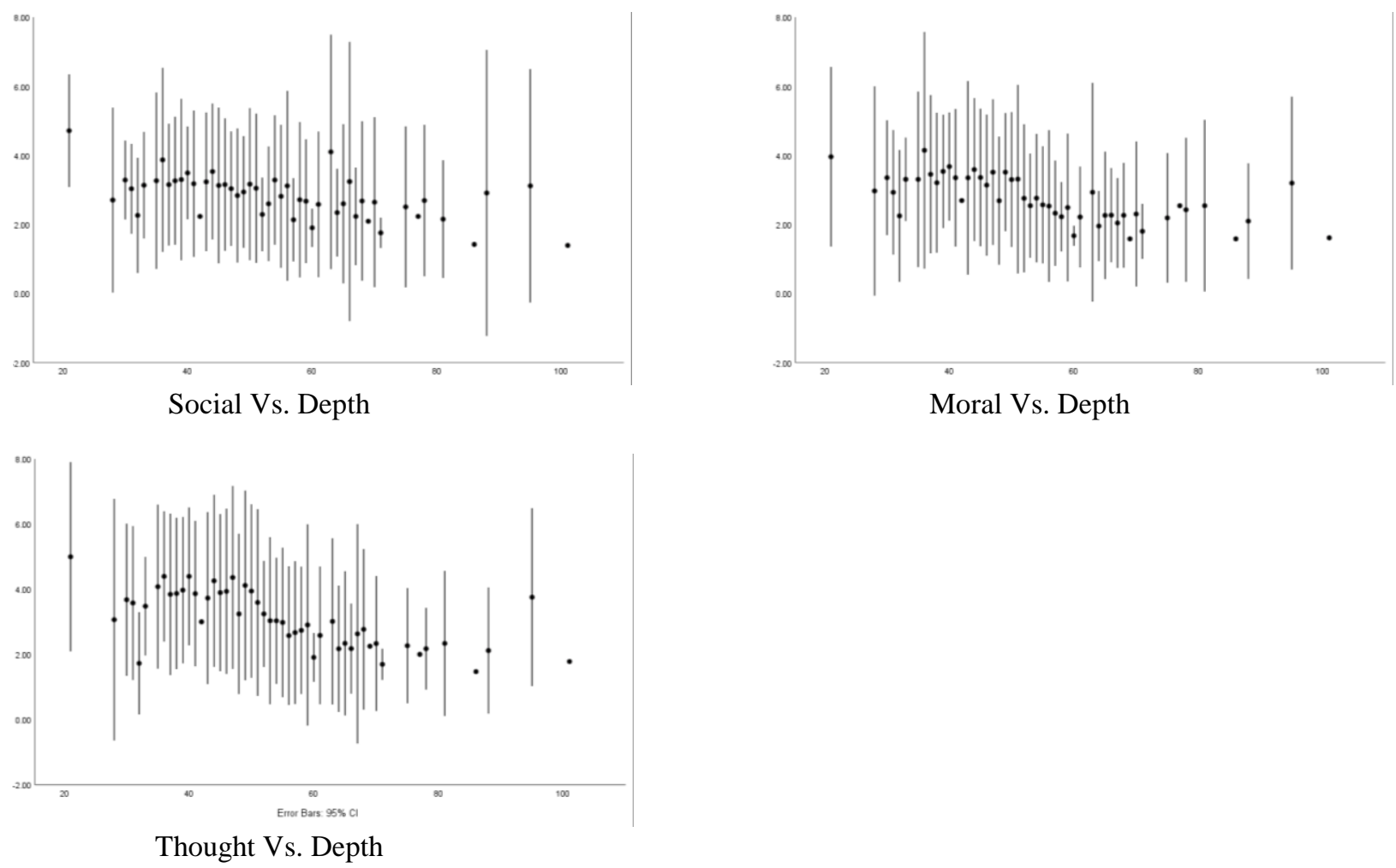

Figure 4. Scatter Plots of Mean Social, Moral and Thought Scores measured in Crutch et al. (2013) Vs Binned Depth. Error Bars represent \pm 2 Standard Deviation. 


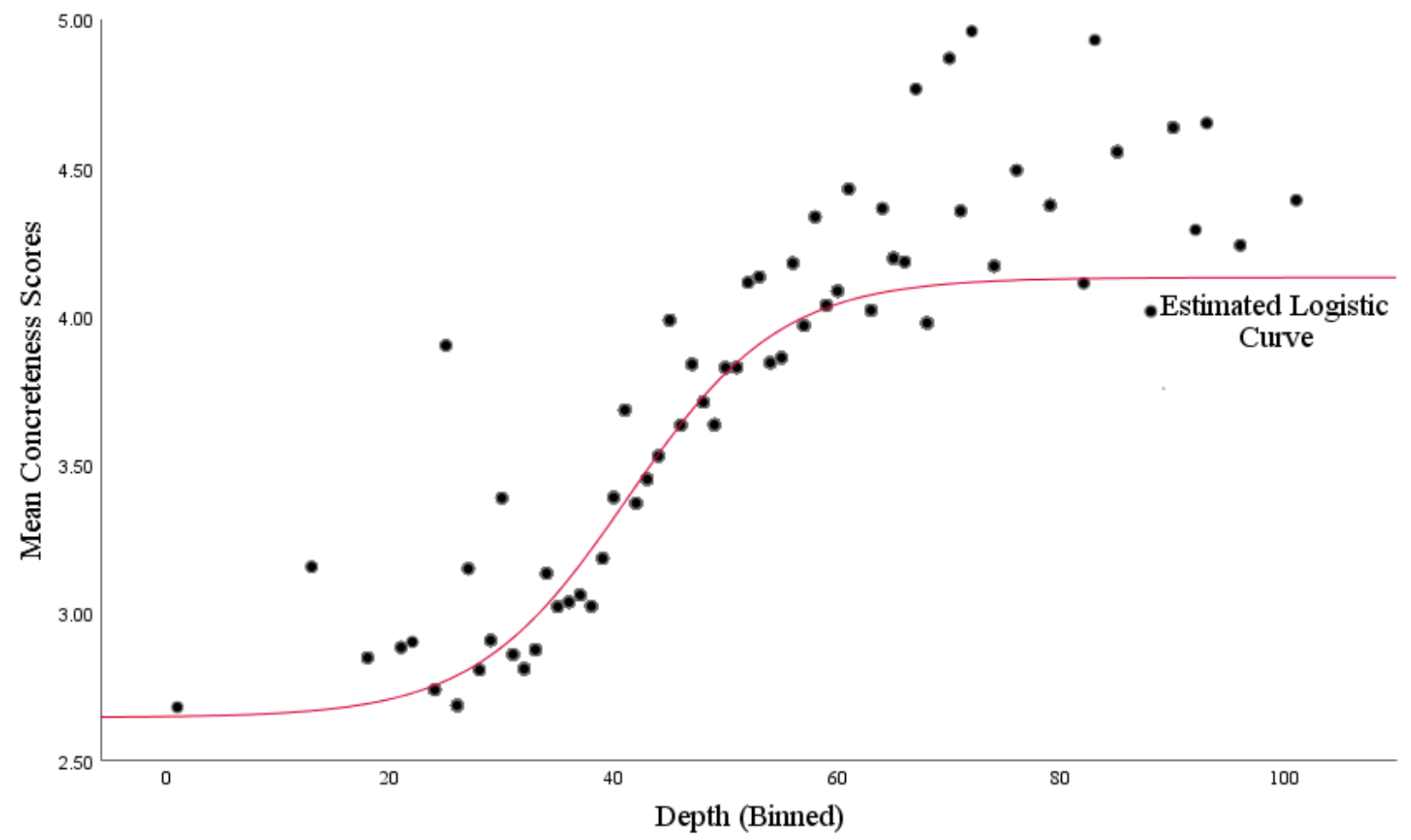

Figure 5. Logistic Regression Function Trained on BYRS Dataset with an Adjusted $\mathrm{R}^{2}=0.939$. The lower and upper asymptotes converge to 2.645 and 4.129 . 


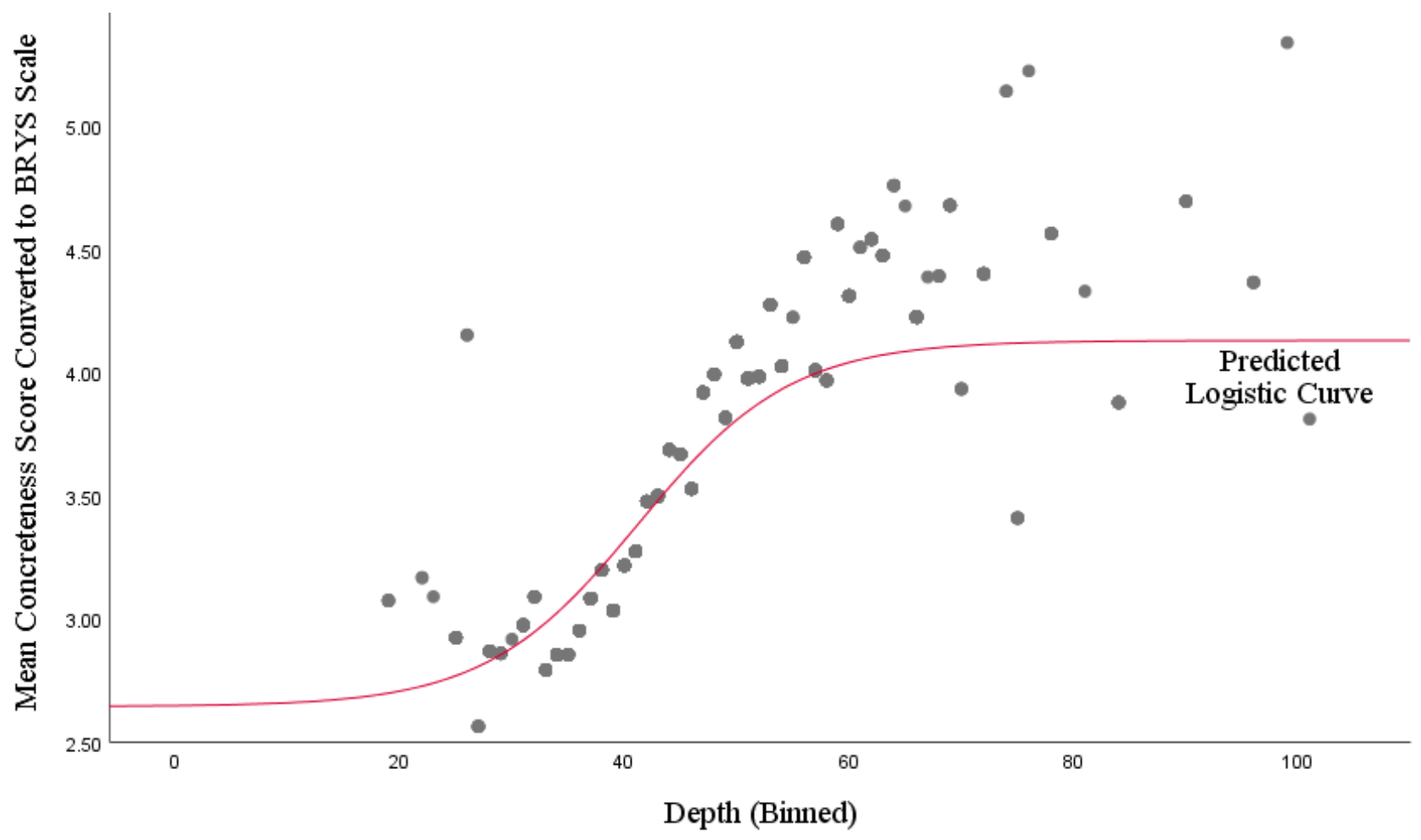

Figure 6. The Logistic Regression Function applied to Converted Concreteness Scores in MRC Dataset. 